\title{
Epsilon-Near-Zero Waveguides for Quantum Information Applications: A Theoretical Approach for N-Qubits
}

\author{
Ege Özgün ${ }^{1}$ and Ekmel Ozbay ${ }^{1,2}$ \\ ${ }^{1}$ NANOTAM-Nanotechnology Research Center, Bilkent University, 06800 Ankara, Turkey \\ ${ }^{2}$ Department of Physics, Department of Electrical and Electronics Engineering, \\ UNAM-Institute of Materials Science and Nanotechnology Bilkent University, 06800 Ankara, Turkey
}

(Received March 1, 2018; accepted September 12, 2018; published online October 22, 2018)

\begin{abstract}
We study the cases of two-, three-, and N-qubits placed inside an epsilon-near-zero (ENZ) waveguide (WG) and present a formalism for the steady-state solution that applies for each case. For equal qubit parameters, we calculate the probability amplitudes of qubits to absorb a photon and make a transition to their excited states. Our results for the $\mathrm{N}$ qubit case can pave the way for multi-qubit entanglement and quantum error correction schemes as well as superradiance studies.
\end{abstract}

\section{Introduction}

Achieving multi-qubit entanglement is necessary for quantum error correction schemes, which require at least tripartite entanglement. In previous studies, by using ions, ${ }^{1)}$ spins, ${ }^{2)}$ photons, ${ }^{3)}$ and a superconducting circuit, ${ }^{4)}$ multiqubit entanglement was demonstrated. Despite these successful attempts, a more accessible scheme is still needed. Although there exists recent theoretical papers investigating two-qubit entanglement in waveguide (WG) geometries, ${ }^{5-9}$ ) their generalisation to the multi-qubit case is not trivial. Another significant application of having multiple emitters is the phenomenon of superradiance, ${ }^{10)}$ in which large number of emitters make spontaneous emission resulting in the significant enhancement of the light intensity. For N-emitters, for instance, the intensity of the light is proportional to $N^{2}$.

Recently, an alternative platform for entanglement generation and manipulation was studied. ${ }^{11)}$ In this study, a photonic crystal (PC) WG was operated at its cut-off frequency yielding an epsilon-near-zero (ENZ) regime. We will base our analytical calculations on that platform, but our calculations in the current manuscript are more general in the sense that our results also apply for plasmonic WGs. In the present paper, we present an analytical solution for $\mathrm{N}$-qubits lying inside an ENZ WG, which would pose an alternative strategy for realising multi-qubit entanglement, quantum error correction schemes and superradiance. In an ENZ medium, the index of the medium approaches zero, resulting in the stretching of the effective wavelength. ${ }^{12)}$ There exists different schemes for obtaining ENZ behaviour. ${ }^{13)}$ Among these, operating the WG near its cut-off is commonly used. We will also utilize this strategy. The advantage of using an ENZ WG is not only the simplifications arising in the analytical calculations; it also shows itself in an experimental point of view, by bringing a huge amount of flexibility to the qubits' positions inside the WG since the phase of the electric field is same at each point for the ENZ region, i.e., the qubits placed inside an ENZ waveguide experience the same interaction, making their placement insensitive to position. This is also crucial for obtaining superradiance, which requires closely spaced emitters. Thanks to the ENZ region we obtain inside the waveguide, it is possible to place a large number of emitters, and due to the spreading of the phase they do not need to be closely spaced now. Recently, enhancement of superradiance in ENZ plasmonic channels was studied. ${ }^{14)}$ The scheme and the calculations we demonstrate throughout this manuscript would also serve as a preliminary study for achieving enhanced superradiance in not only ENZ plasmonic WGs but also for ENZ PC WGs.

Our theoretical approach is based on the previous calculations for WG-quantum dot coupled systems. ${ }^{15)} \mathrm{We}$ extend it to the cases of three- and $\mathrm{N}$-qubits. The significant difference in our calculations is the simplifications arising thanks to the ENZ behaviour of the WG. We will neglect the direct dipole-dipole interactions by assuming that the qubits are placed distant enough so that the only interaction between them is the one mediated by the photons inside the WG.

Firstly, we demonstrate the numerical results in Sect. 2 for the ENZ WG, which is essential for our mathematical model. Then we start introducing our model with the two-qubit case in Sect. 3 and extend the same formalism to the three-qubit case in Sect. 4. Then, we generalize our results to the $\mathrm{N}$-qubit case in Sect. 5. In Sect. 6 several possible applications are presented. We conclude with Sect. 7.

\section{Numerical Results for the ENZ WG}

Achieving ENZ behaviour is necessary for our model. For this study, it can be either a PC WG or a plasmonic WG: the essential part is attaining the ENZ regime. Figures 1 and 2 present the results of the FDTD calculations performed with the commercial software Lumerical for an InP PC WG. The magnitudes (Fig. 1) and phases (Fig. 2) for the electric field (E-field) profiles are given for different wavelengths. The constant phase inside the WG is one of the clear signatures of the ENZ regime. The ENZ behaviour is obtained for the wavelength $\lambda=1.49477 \mu \mathrm{m}$ which corresponds to the cut-off frequency of the WG. This value can be tuned by changing the width of the WG but it is out of the scope of the current study. As we move away from the cut-off, ENZ behaviour vanishes, as it can be seen from Figs. 1 and 2. Throughout this paper, we will assume that we are working in the ENZ regime, hence for a wide range of qubit separations (d), $k \ll 1 / d$ is satisfied ( $k$ being the wavenumber of the guiding mode), which both yields mathematical simplifications and experimental ease in placement of the qubits. 

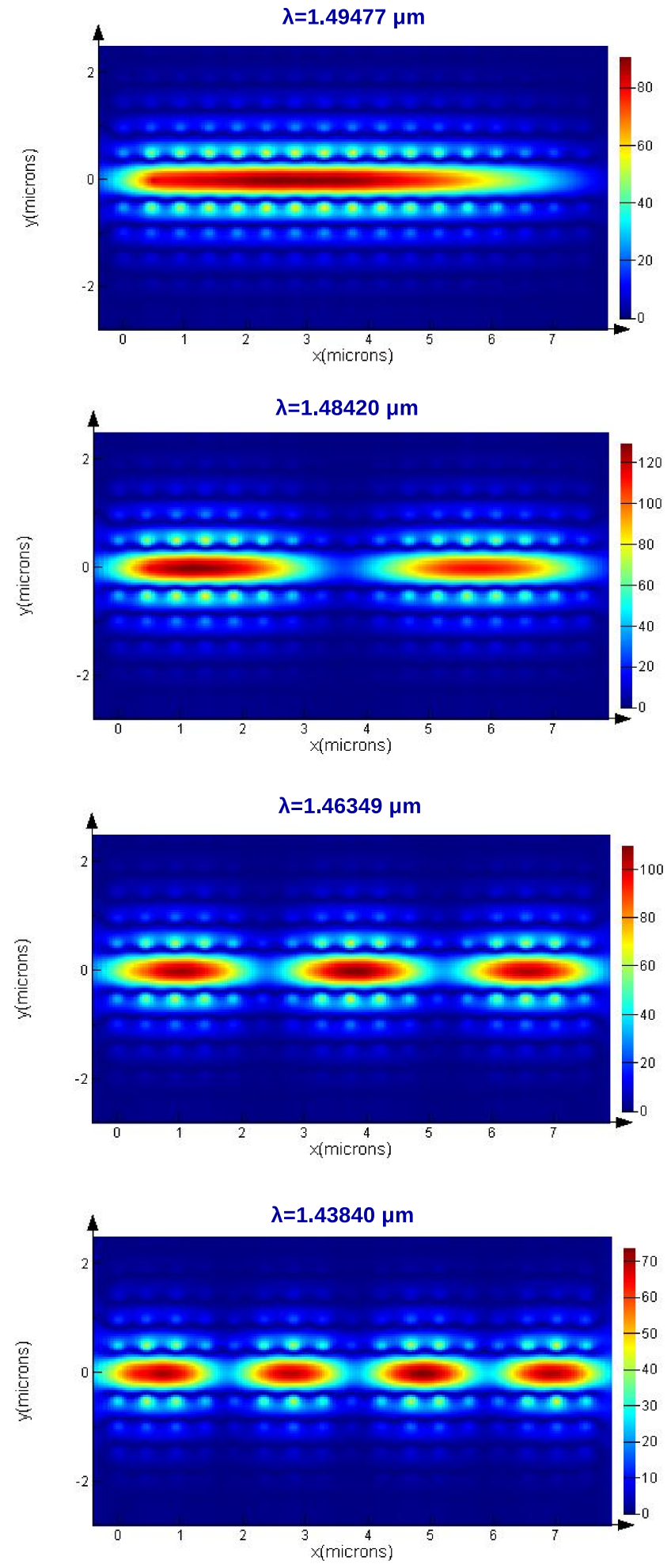

Fig. 1. (Color online) Magnitudes of the E-fields given in $(\mathrm{V} / \mathrm{m})$ for different wavelengths are illustrated. $\lambda=1.49477 \mu \mathrm{m}$ case gives the ENZ regime, that is obtained by operating the WG near its cut-off frequency. As the wavelength is decreased, the ENZ behaviour vanishes.

\section{Two-qubit Case}

Qubit positions for each three cases, and the graphical illustration of the qubits lying inside the WG is given in Fig. 3. We will start with the two-qubit case. Although it was already studied within the ENZ context, ${ }^{16)}$ it is instructive to introduce our formalism starting from the simplest case. Our starting point is the Jaynes-Cummings
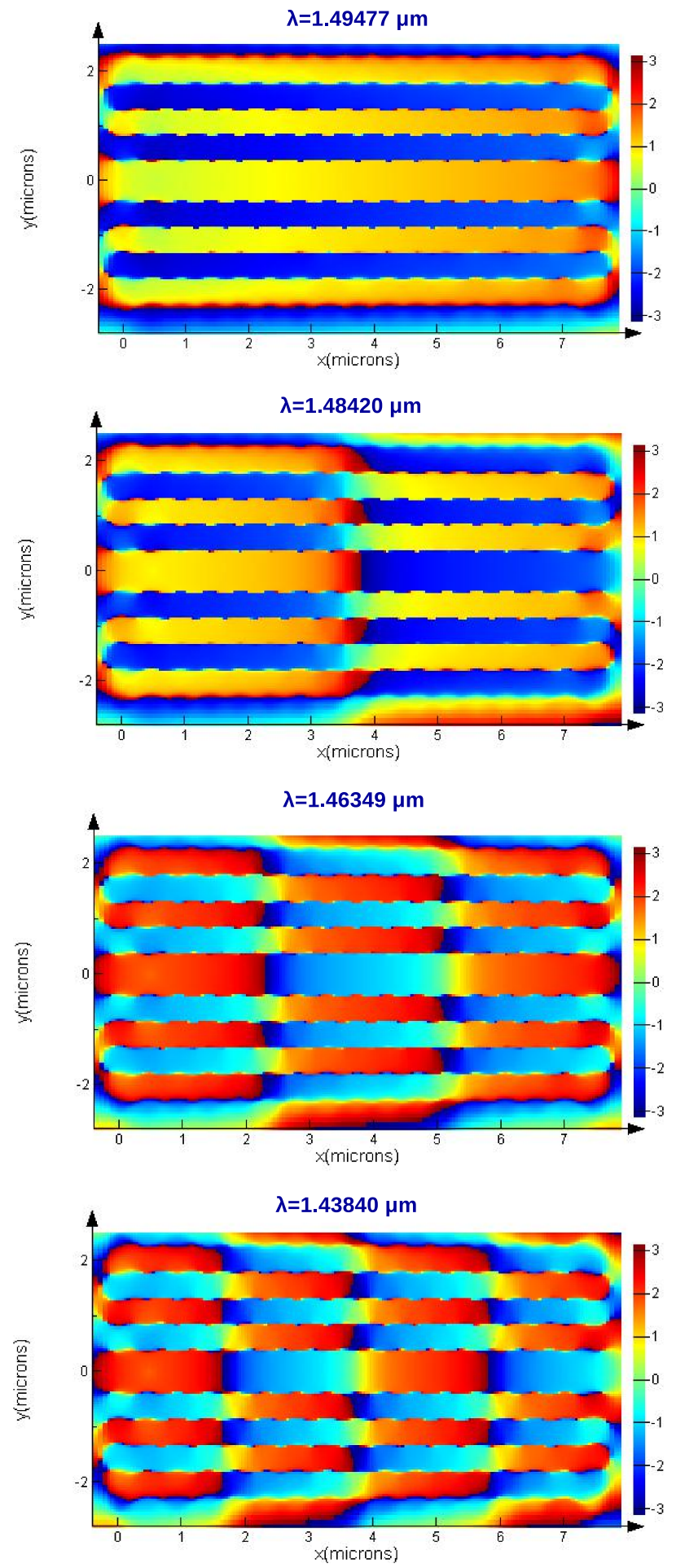

Fig. 2. (Color online) Same as Fig. 1, but Phases of the E-fields for different wavelengths are illustrated.

Hamiltonian in the rotating wave approximation in which we take $\hbar=1$,

$$
\begin{aligned}
\hat{H}_{2}= & \sum_{k}\left[\omega_{k} c_{k}^{\dagger} c_{k}+\sum_{j=1}^{2} V_{k}^{(j)}\left(c_{k}^{\dagger} \sigma_{-}^{(j)}+c_{k} \sigma_{+}^{(j)}\right)\right] \\
& +\sum_{j=1}^{2} \sigma_{+}^{(j)} \sigma_{-}^{(j)}\left(\omega_{j}-i \frac{\Gamma_{j}}{2}\right),
\end{aligned}
$$

where $\omega_{k}$ is the frequency of the waveguide mode with wavenumber $\mathrm{k}, V_{k}^{(j)}$, s are the k-dependent photon-qubit 
a)

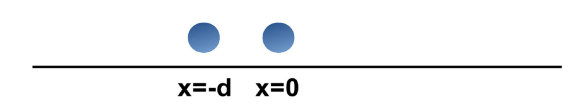

b)

$x=-2 d \quad x=-d \quad x=0$

c)

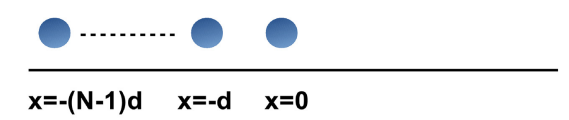

d)

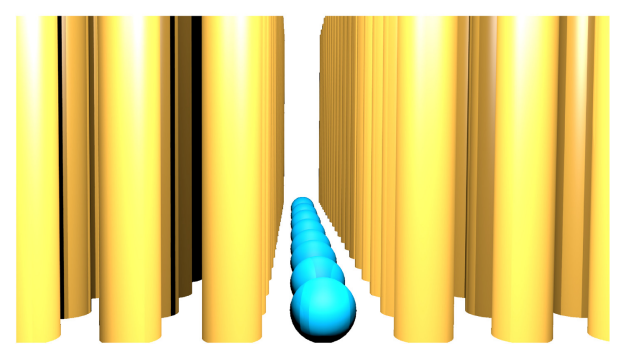

Fig. 3. (Color online) Sketch of qubit positions and separations for (a) two-qubit (b) three-qubit and (c) N-qubit cases and graphical illustration of qubits lying inside the PC WG (d).

interaction strengths, $\omega_{j}$ 's and $\Gamma_{j}$ 's are the excitonic transition frequencies and losses of the qubits, $c_{k}^{\dagger} / c_{k}$ are bosonic creation/annihilation operators, and $\sigma_{+}^{(j)} / \sigma_{-}^{(j)}$ are raising/lowering operators for the qubits. We can linearise the above Hamiltonian and recast it in real-space (the linearisation of the dispersion starts losing its validity in the close vicinity of the cut-off frequency of the WG and, therefore, we choose a value that both avoids this regime, and at the same time, yields the desired ENZ behaviour). In doing so, we have:

$$
\begin{aligned}
\hat{\mathcal{H}}_{2}= & \int d x\left\{c_{R}^{\dagger}(x)\left(\omega_{0}-i v_{g} \frac{\partial}{\partial x}\right) c_{R}(x)\right. \\
& +c_{L}^{\dagger}(x)\left(\omega_{0}+i v_{g} \frac{\partial}{\partial x}\right) c_{L}(x) \\
& +\sum_{j=1}^{2} g_{j} \delta(x-[j-2] d)\left[c_{R}^{\dagger}(x) \sigma_{-}^{(j)}\right. \\
& \left.\left.+c_{R}(x) \sigma_{+}^{(j)}+c_{L}^{\dagger}(x) \sigma_{-}^{(j)}+c_{L}(x) \sigma_{+}^{(j)}\right]\right\} \\
& +\sum_{j=1}^{2} \sigma_{+}^{(j)} \sigma_{-}^{(j)}\left(\omega_{j}-i \frac{\Gamma_{j}}{2}\right)
\end{aligned}
$$

in which $\omega_{0}$ is the frequency of the single mode that we are operating at, $v_{g}$ is the group velocity of the photon inside the WG, $g_{j}$ 's are the photon-qubit interaction strengths for the single mode, $d$ is the qubit separation and $c_{R}^{\dagger}(x) / c_{R}(x)\left[c_{L}^{\dagger}(x) /\right.$ $\left.c_{L}(x)\right]$ are the bosonic creation/annihilation operators for the right[left]-going modes. We can write the time-independent solution consisting of three states: One of the qubits can absorb the photon and make a transition to its excited state $\left(|0\rangle_{p} \otimes\left|e_{1}, g_{2}\right\rangle\right.$ or $\left.|0\rangle_{p} \otimes\left|g_{1}, e_{2}\right\rangle\right)$ or the photon can propagate inside the WG $\left(|1\rangle_{p} \otimes\left|g_{1}, g_{2}\right\rangle\right)$. We can calculate the steady-state solution by solving the Schrödinger equation $\hat{\mathcal{H}}_{2} \Psi_{2}=\varepsilon \Psi_{2}$, where $\varepsilon=w_{0}$ and the wavefunction is given by the following:

$$
\begin{aligned}
\left|\Psi_{2}\right\rangle= & \int d x\left[\phi_{R}^{(2)}(x) c_{R}^{\dagger}(x)+\phi_{L}^{(2)}(x) c_{L}^{\dagger}(x)\right]|0\rangle_{p} \otimes\left|g_{1}, g_{2}\right\rangle \\
& +\alpha_{1}|0\rangle_{p} \otimes\left|e_{1}, g_{2}\right\rangle+\alpha_{2}|0\rangle_{p} \otimes\left|g_{1}, e_{2}\right\rangle
\end{aligned}
$$

Here $\alpha_{1}$ and $\alpha_{2}$ are the probability amplitudes for the qubits to make a transition to their excited states by absorbing the photon and the $\phi_{R}^{(2)}(x) / \phi_{L}^{(2)}(x)$ are the scattering amplitudes:

$$
\begin{aligned}
& \phi_{R}^{(2)}(x)=e^{i k x}[\theta(-x-d)+a \theta(x+d) \theta(-x)+t \theta(x)], \\
& \phi_{L}^{(2)}(x)=e^{-i k x}[r \theta(-x-d)+b \theta(x+d) \theta(-x)],
\end{aligned}
$$

where $t / r$ are the transmission/reflection coefficients and $a$ and $b$ are the probability amplitudes for the photon to be found between two qubits. We can now solve the eigenvalue problem to obtain the following equations:

$$
\begin{aligned}
g_{1}\left[e^{i k d}(r+b)+e^{-i k d}(1+a)\right] & =\alpha_{1}\left[\Delta_{1}+i \frac{\Gamma_{1}}{2}\right], \\
g_{2}[a+t+b] & =\alpha_{2}\left[\Delta_{2}+i \frac{\Gamma_{2}}{2}\right], \\
i v_{g}[t-a] & =g_{2} \alpha_{2}, \\
i v_{g}[a-1] e^{-i k d} & =g_{1} \alpha_{1}, \\
i v_{g} b & =g_{2} \alpha_{2}, \\
i v_{g}[r-b] e^{i k d} & =g_{1} \alpha_{1} .
\end{aligned}
$$

Here, we defined $\Delta_{j} \equiv \omega_{0}-\omega_{j}$. Now, we will exploit the ENZ behaviour of the WG: As long as $k \ll 1 / d$, we can replace the exponential terms in Eq. (4) with unity. For an ENZ WG we can safely apply this approximation. Solving for $t, r, a$, and $b$, plugging them in Eqs. (4a), (4b) and taking equal values for the qubit parameters $\left.{ }^{17}\right)\left(\Delta_{1}=\Delta_{2} \equiv \Delta\right.$, $\Gamma_{1}=\Gamma_{2} \equiv \Gamma$, and $g_{1}=g_{2} \equiv g$ ), we arrive at two equations to be solved for $\alpha_{1}$ and $\alpha_{2}$ :

$$
\begin{aligned}
& \left(\frac{\Delta+i \frac{\Gamma}{2}}{2 g}-\frac{g}{i v_{g}}\right) \alpha_{1}-\frac{g}{i v_{g}} \alpha_{2}=1, \\
& \left(\frac{\Delta+i \frac{\Gamma}{2}}{2 g}-\frac{g}{i v_{g}}\right) \alpha_{2}-\frac{g}{i v_{g}} \alpha_{1}=1 .
\end{aligned}
$$

We can rewrite Eq. (5) in matrix from as $\mathbf{M} \boldsymbol{\alpha}=\boldsymbol{C}_{2}$ (The necessity of this step will be revealed when we are investigating the $\mathrm{N}$-qubit case in Sect. 5.) where, 


$$
\mathbf{M}=\left(\begin{array}{cc}
x-y & -y \\
-y & x-y
\end{array}\right), \quad \boldsymbol{\alpha}=\left(\begin{array}{l}
\alpha_{1} \\
\alpha_{2}
\end{array}\right), \quad \boldsymbol{C}_{2}=\left(\begin{array}{l}
1 \\
1
\end{array}\right)
$$

in which $x=(\Delta+i \Gamma / 2) / 2 g$ and $y=g / i v_{g}$. Now, we can easily write the solution for $\boldsymbol{\alpha}$ as:

$$
\boldsymbol{\alpha}=\mathbf{M}^{-1} \boldsymbol{C}_{2}
$$

Finally, calculating the inverse of $\mathbf{M}$ we have:

$$
\begin{aligned}
\mathbf{M}^{-1} & =\frac{1}{2 x y-x^{2}}\left(\begin{array}{cc}
y-x & -y \\
-y & y-x
\end{array}\right), \\
\alpha_{1}=\alpha_{2} & =\frac{1}{2 x y-x^{2}}[y-x-y] \\
& =\left[\frac{\Delta+i \frac{\Gamma}{2}}{2 g}-\frac{2 g}{i v_{g}}\right]^{-1} .
\end{aligned}
$$

As one would naturally expect, $\alpha_{1}$ and $\alpha_{2}$ are equal, since we have chosen the same values for the qubit parameters. Let us move on to the three-qubit case, in which the pattern for the $\mathrm{N}$-qubit case will start showing itself.

\section{Three-qubit Case}

We again cast the Jaynes-Cummings Hamiltonian, but this time we have three-qubits.

$$
\begin{aligned}
\hat{H}_{3}= & \sum_{k}\left[\omega_{k} c_{k}^{\dagger} c_{k}+\sum_{j=1}^{3} V_{k}^{(j)}\left(c_{k}^{\dagger} \sigma_{-}^{(j)}+c_{k} \sigma_{+}^{(j)}\right)\right] \\
& +\sum_{j=1}^{3} \sigma_{+}^{(j)} \sigma_{-}^{(j)}\left(\omega_{j}-i \frac{\Gamma_{j}}{2}\right)
\end{aligned}
$$

Following the same procedure of Sect. 3, we can write the real-space Hamiltonian by linearising the dispersion:

$$
\begin{aligned}
\hat{\mathcal{H}}_{3}= & \int d x\left\{c_{R}^{\dagger}(x)\left(\omega_{0}-i v_{g} \frac{\partial}{\partial x}\right) c_{R}(x)\right. \\
& +c_{L}^{\dagger}(x)\left(\omega_{0}+i v_{g} \frac{\partial}{\partial x}\right) c_{L}(x) \\
& +\sum_{j=1}^{3} g_{j} \delta(x-[j-3] d)\left[c_{R}^{\dagger}(x) \sigma_{-}^{(j)}\right. \\
& \left.\left.+c_{R}(x) \sigma_{+}^{(j)}+c_{L}^{\dagger}(x) \sigma_{-}^{(j)}+c_{L}(x) \sigma_{+}^{(j)}\right]\right\} \\
& +\sum_{j=1}^{3} \sigma_{+}^{(j)} \sigma_{-}^{(j)}\left(\omega_{j}-i \frac{\Gamma_{j}}{2}\right) .
\end{aligned}
$$

Our time-independent solution for the three-qubit case follows the same logic: The photon can be absorbed by one of the three-qubits, causing them to make a transition to their excited states or the photon can propagate inside the WG.

$$
\begin{aligned}
\left|\Psi_{3}\right\rangle= & \int d x\left[\phi_{R}^{(3)}(x) c_{R}^{\dagger}(x)+\phi_{L}^{(3)}(x) c_{L}^{\dagger}(x)\right]|0\rangle_{p} \otimes\left|g_{1}, g_{2}, g_{3}\right\rangle \\
& +\alpha_{1}|0\rangle_{p} \otimes\left|e_{1}, g_{2}, g_{3}\right\rangle+\alpha_{2}|0\rangle_{p} \otimes\left|g_{1}, e_{2}, g_{3}\right\rangle \\
& +\alpha_{3}|0\rangle_{p} \otimes\left|g_{1}, g_{2}, e_{3}\right\rangle
\end{aligned}
$$

$$
\begin{aligned}
\phi_{R}^{(3)}(x)= & e^{i k x}\left[\theta(-x-2 d)+a_{1} \theta(x+2 d) \theta(-x+d)\right. \\
& \left.+a_{2} \theta(x-d) \theta(-x)+t \theta(x)\right], \\
\phi_{L}^{(3)}(x)= & e^{-i k x}\left[r \theta(-x-2 d)+b_{1} \theta(x+2 d) \theta(-x+d)\right. \\
& \left.+b_{2} \theta(x-d) \theta(-x)\right] .
\end{aligned}
$$

After solving the Schrödinger equation, $\hat{\mathcal{H}}_{3} \Psi_{3}=\varepsilon \Psi_{3}$, this time we end up with nine coupled equations instead of six. After choosing equal values for the qubit parameters and replacing the exponential terms with unity we have the following equations for $\alpha_{1}, \alpha_{2}$, and $\alpha_{3}$ :

$$
\begin{aligned}
& \left(\frac{\Delta+i \frac{\Gamma}{2}}{2 g}-\frac{g}{i v_{g}}\right) \alpha_{1}-\frac{g}{i v_{g}}\left[\alpha_{2}+\alpha_{3}\right]=1, \\
& \left(\frac{\Delta+i \frac{\Gamma}{2}}{2 g}-\frac{g}{i v_{g}}\right) \alpha_{2}-\frac{g}{i v_{g}}\left[\alpha_{1}+\alpha_{3}\right]=1, \\
& \left(\frac{\Delta+i \frac{\Gamma}{2}}{2 g}-\frac{g}{i v_{g}}\right) \alpha_{3}-\frac{g}{i v_{g}}\left[\alpha_{1}+\alpha_{2}\right]=1 .
\end{aligned}
$$

Just like the two-qubit case, we can express the equations for $\alpha$ 's in matrix form, $\mathbf{M} \boldsymbol{\alpha}=\boldsymbol{C}_{3}$, in which,

$$
\begin{gathered}
\mathbf{M}=\left(\begin{array}{ccc}
x-y & -y & -y \\
-y & x-y & -y \\
-y & -y & x-y
\end{array}\right), \\
\boldsymbol{\alpha}=\left(\begin{array}{l}
\alpha_{1} \\
\alpha_{2} \\
\alpha_{3}
\end{array}\right), \quad \boldsymbol{C}_{3}=\left(\begin{array}{l}
1 \\
1 \\
1
\end{array}\right) .
\end{gathered}
$$

Again, we calculate the inverse of $\mathbf{M}$ to obtain $\boldsymbol{\alpha}$ :

$$
\begin{aligned}
\mathbf{M}^{-1} & =\frac{1}{3 x y-x^{2}}\left(\begin{array}{ccc}
2 y-x & -y & -y \\
-y & 2 y-x & -y \\
-y & -y & 2 y-x
\end{array}\right), \\
\alpha_{1} & =\alpha_{2}=\alpha_{3}=\frac{1}{3 x y-x^{2}}[2 y-x-y-y] \\
& =\left[\frac{\Delta+i \frac{\Gamma}{2}}{2 g}-\frac{3 g}{i v_{g}}\right]^{-1} .
\end{aligned}
$$

Equations (7) and (12) already reveal the pattern we are seeking, but let us present the same formalism for the $\mathrm{N}$-qubit case starting from the beginning.

\section{N-qubit Case}

We are now in a position to present the analytical solution for the N-qubit case. The Jaynes-Cummings Hamiltonian for $\mathrm{N}$-qubits reads:

$$
\hat{H}_{N}=\sum_{k}\left[\omega_{k} c_{k}^{\dagger} c_{k}+\sum_{j=1}^{N} V_{k}^{(j)}\left(c_{k}^{\dagger} \sigma_{-}^{(j)}+c_{k} \sigma_{+}^{(j)}\right)\right]
$$

in which the scattering amplitudes are given by, 


$$
+\sum_{j=1}^{N} \sigma_{+}^{(j)} \sigma_{-}^{(j)}\left(\omega_{j}-i \frac{\Gamma_{j}}{2}\right) .
$$

We follow the same procedure and recast the Hamiltonian in real-space,

$$
\begin{aligned}
\hat{\mathcal{H}}_{N}= & \int d x\left\{c_{R}^{\dagger}(x)\left(\omega_{0}-i v_{g} \frac{\partial}{\partial x}\right) c_{R}(x)\right. \\
& +c_{L}^{\dagger}(x)\left(\omega_{0}+i v_{g} \frac{\partial}{\partial x}\right) c_{L}(x) \\
& +\sum_{j=1}^{N} g_{j} \delta(x-[j-N] d)\left[c_{R}^{\dagger}(x) \sigma_{-}^{(j)}\right. \\
& \left.\left.+c_{R}(x) \sigma_{+}^{(j)}+c_{L}^{\dagger}(x) \sigma_{-}^{(j)}+c_{L}(x) \sigma_{+}^{(j)}\right]\right\} \\
& +\sum_{j=1}^{N} \sigma_{+}^{(j)} \sigma_{-}^{(j)}\left(\omega_{j}-i \frac{\Gamma_{j}}{2}\right) .
\end{aligned}
$$

For $\mathrm{N}$-qubits, we have $\mathrm{N}+1$ terms in our steady state solution. One term for the photon propagating inside the WG and n-terms describing the probability that any one of the $\mathrm{N}$-qubits absorbing a photon and making a transition to its excited state:

$$
\begin{aligned}
\left|\Psi_{N}\right\rangle= & \int d x\left[\phi_{R}^{(N)}(x) c_{R}^{\dagger}(x)+\phi_{L}^{(N)}(x) c_{L}^{\dagger}(x)\right]|0\rangle_{p} \\
& \otimes\left|g_{1}, g_{2}, \ldots, g_{N}\right\rangle+\alpha_{1}|0\rangle_{p} \otimes\left|e_{1}, g_{2}, \ldots, g_{N}\right\rangle \\
& +\alpha_{2}|0\rangle_{p} \otimes\left|g_{1}, e_{2}, \ldots, g_{N}\right\rangle+\cdots \\
& +\alpha_{N}|0\rangle_{p} \otimes\left|g_{1}, g_{2}, \ldots, e_{N}\right\rangle
\end{aligned}
$$

with the scattering amplitudes,

$$
\begin{aligned}
\phi_{R}^{(N)}(x)= & e^{i k x}[\theta(-x-(N-1) d) \\
& +a_{1} \theta(x+(N-1) d) \theta(-x+(N-2) d) \\
& +a_{2} \theta(x-(N-2) d) \theta(-x+(N-3) d)+\cdots \\
& \left.+a_{N} \theta(x-d) \theta(-x)+t \theta(x)\right], \\
\phi_{L}^{(N)}(x)= & e^{-i k x}[r \theta(-x-(N-1) d) \\
& +b_{1} \theta(x+(N-1) d) \theta(-x+(N-2) d) \\
& +b_{2} \theta(x-(N-2) d) \theta(-x+(N-3) d)+\cdots \\
& \left.+b_{N} \theta(x-d) \theta(-x)\right] .
\end{aligned}
$$

The number of equations we have for $\mathrm{N}$-qubits is $3 n: 2 n$ of them are needed to express $r, t, a_{1}, a_{2}, \ldots, a_{N}$ and $b_{1}, b_{2}, \ldots$, $b_{N}$ in terms of $\alpha_{1}, \alpha_{2}, \ldots, \alpha_{N}$ and the remaining $n$ are the equations for $\alpha_{1}, \alpha_{2}, \ldots, \alpha_{N}$. Following the same steps, the equations for $\alpha$ 's are found to be:

$$
\begin{aligned}
& \left(\frac{\Delta+i \frac{\Gamma}{2}}{2 g}-\frac{g}{i v_{g}}\right) \alpha_{1}-\frac{g}{i v_{g}}\left[\alpha_{2}+\alpha_{3}+\cdots+\alpha_{N}\right]=1 \\
& \left(\frac{\Delta+i \frac{\Gamma}{2}}{2 g}-\frac{g}{i v_{g}}\right) \alpha_{2}-\frac{g}{i v_{g}}\left[\alpha_{1}+\alpha_{3}+\cdots+\alpha_{N}\right]=1 \\
& \left(\frac{\Delta+i \frac{\Gamma}{2}}{2 g}-\frac{g}{i v_{g}}\right) \alpha_{N-1}-\frac{g}{i v_{g}}\left[\alpha_{1}+\alpha_{2}+\cdots+\alpha_{N-2}+\alpha_{N}\right] \\
& =1 \\
& \left(\frac{\Delta+i \frac{\Gamma}{2}}{2 g}-\frac{g}{i v_{g}}\right) \alpha_{N}-\frac{g}{i v_{g}}\left[\alpha_{1}+\alpha_{2}+\cdots+\alpha_{N-1}\right]=1 .
\end{aligned}
$$

We again express the equations for $\alpha$ 's in matrix form, $\mathbf{M} \boldsymbol{\alpha}=\boldsymbol{C}_{N}$, where,

$$
\begin{gathered}
\mathbf{M}=\left(\begin{array}{ccccc}
x-y & -y & \ldots & \ldots & -y \\
-y & x-y & \ddots & \ddots & \vdots \\
\vdots & \ddots & \ddots & \ddots & \vdots \\
\vdots & \ddots & \ddots & \ddots & -y \\
-y & \ldots & \ldots & -y & x-y
\end{array}\right), \\
\boldsymbol{\alpha}
\end{gathered}
$$

The inverse of $\mathbf{M}$ and $\boldsymbol{\alpha}$ for the N-qubit case read:

$$
\begin{aligned}
& \mathbf{M}^{-1}=\frac{1}{N x y-x^{2}}\left(\begin{array}{ccccc}
(N-1) y-x & -y & \cdots & \cdots & -y \\
-y & (N-1) y-x & \ddots & \ddots & \vdots \\
\vdots & \ddots & \ddots & \ddots & \vdots \\
\vdots & \ddots & \ddots & \ddots & -y \\
-y & \ldots & \ldots & -y & (N-1) y-x
\end{array}\right) \\
& \alpha_{1}=\alpha_{2}=\cdots=\alpha_{N}=\frac{1}{N x y-x^{2}}[(N-1) y-x-\underbrace{y-y-\cdots-y}_{N-1}] \\
& =\left[\frac{\Delta+i \frac{\Gamma}{2}}{2 g}-\frac{N g}{i v_{g}}\right]^{-1} .
\end{aligned}
$$




\section{Possible Applications}

There are three possible main applications that can be based on our calculations for N-qubits. It is important to note that our suggested scheme and the results of our calculations are valid for both PC and plasmonic WGs, as long as the WG is operated near its cut-off to yield ENZ behaviour.

The first two possible application are realising multi-qubit entanglement and quantum error correction schemes. The placement of qubits and fabrication and implementation of the platform are the main challenges. Our scheme, which was based on our previous work, ${ }^{11)}$ but is also valid for plasmonic WGs, overcomes both difficulties. It eliminates the difficulty in qubit placement thanks to the spreading of the phase due to the ENZ regime we work in. Moreover, the scheme we use for our calculations is realisable in real life and more advantageous compared to the other methods for multi-qubit entanglement since fabrication and manipulation of plasmonic and PC WGs are more reachable than the other schemes suggested.

The third and last possible application is the enhancement of superradiance. For achieving the collective spontaneous emission of emitters, effectively the wavelength of the light have to be larger than the separation of the emitters. In an ENZ medium this condition is automatically satisfied, since all the emitters will feel the same interaction due to the spreading of the phase. Therefore our scheme and calculations can be used to realise the previous studies on enhanced superradiance in ENZ plasmonic WGs, ${ }^{14)}$ and also to realise enhanced superradiance in ENZ PC WGs.

\section{Conclusions}

In summary, we presented an analytical study of $\mathrm{N}$-qubits lying inside an ENZ WG. By assuming equal qubit parameters, we derived the probability amplitudes of qubits to absorb a photon and make a transition to their excited states. Our suggested scheme, formalism and calculations will be significant for multi-qubit entanglement and quantumerror correction schemes and superradiance studies that utilise ENZ WGs. A theoretical foundation, moreover a fully analytical calculation for multi-qubit entanglement, i.e., for multi-qubit entanglement generation is a significant step in understanding and realizing error correction schemes for multi-qubit quantum computing systems. The introduction of ENZ properties for quantum computing possesses great advantages and our fully analytical theoretical model poses a good starting point for this more realizable quantum computing scheme.

Acknowledgement This work is supported by the projects DPT-HAMIT and NATO-SET-193. One of the authors (E.O.) also acknowledges partial support from the Turkish Academy of Sciences.

1) H. Häffner, W. Hänsel, C. F. Roos, J. Benhelm, D. Chek-al-kar, M Chwalla, T. Körber, U. D. Rapol, M. Riebe, P. O. Schmidt, C. Becher, O. Gühne, W. Dür, and R. Blatt, Nature 438, 643 (2005).

2) P. Neumann, N. Mizuochi, F. Rempp, P. Hemmer, H. Watanabe, S. Yamasaki, V. Jacques, T. Gaebel, F. Jelezko, and J. Wrachtrup, Science 320, 1326 (2008)

3) W.-B. Gao, C.-Y. Lu, X.-C. Yao, P. Xu, O. Gühne, A. Goebel, Y.-A Chen, C.-Z. Peng, Z.-B. Chen, and J.-W. Pan, Nat. Phys. 6, 331 (2010).

4) L. DiCarlo, M. D. Reed, L. Sun, B. R. Johnson, J. M. Chow, J. M. Gambetta, L. Frunzio, S. M. Girvin, M. H. Devoret, and R. J. Schoelkopf, Nature 467, 574 (2010).

5) A. Gonzalez-Tudela, D. Martin-Cano, E. Moreno, L. Martin-Moreno, C. Tejedor, and F. J. Garcia-Vidal, Phys. Rev. Lett. 106, 020501 (2011).

6) D. Martín-Cano, A. González-Tudela, L. Martín-Moreno, F. J. GarcíaVidal, C. Tejedor, and E. Moreno, Phys. Rev. B 84, 235306 (2011).

7) C. Gonzalez-Ballestero, E. Moreno, and F. J. Garcia-Vidal, Phys. Rev. A 89, 042328 (2014).

8) H. Zheng and H. U. Baranger, Phys. Rev. Lett. 110, 113601 (2013).

9) Z. Liao, X. Zeng, S.-Y. Zhu, and M. Suhail Zubairy, Phys. Rev. A 92, 023806 (2015).

10) R. H. Dicke, Phys. Rev. 93, 99 (1954).

11) E. Özgün, E. Ozbay, and H. Caglayan, ACS Photonics 3, 2129 (2016).

12) M. G. Silveirinha and N. Engheta, Phys. Rev. Lett. 97, 157403 (2006).

13) N. Engheta, Science 340, 286 (2013).

14) R. Fleury and A. Alù, Phys. Rev. B 87, 201101(R) (2013).

15) J. T. Shen and S. Fan, Opt. Lett. 30, 2001 (2005); J. T. Shen and S. Fan, Phys. Rev. Lett. 95, 213001 (2005); J. T. Shen and S. Fan, Phys. Rev. Lett. 98, 153003 (2007); J. T. Shen and S. Fan, Phys. Rev. A 79, 023837 (2009).

16) X. R. Jin, L. Sun, X. Yang, and J. Gao, Opt. Lett. 38, 4078 (2013).

17) Although the main reason behind this choice is mathematical manipulation, for obtaining the maximum amount of entanglement in such a system, equal qubit parameters are required. Therefore, our choice is also realistic for quantum information applications, in which the maximum degree of entanglement is necessary. 